\title{
Formation and Topology of vortices in Couette Flow over open cavities
}

\author{
Cesare Biserni ${ }^{1}$, Andrea Natale Impiombato ${ }^{1}$, Aminhossein Jahanbin ${ }^{1}$, Eugenia Rossi di \\ Schio $^{1 *}$ and Giovanni Semprini ${ }^{1}$ \\ ${ }^{1}$ Department of Industrial Engineering, Alma Mater Studiorum - University of Bologna, Viale \\ Risorgimento 2, 40136 Bologna, Italy.
}

\begin{abstract}
The present study investigates the planar Couette flow problem for low Reynolds numbers inside a rectangular duct with a morphing cavity serving as a vortex formation promoter. A finite element code implemented in COMSOL Multiphysics is employed to analyze the effects of the cavity aspect ratio and variations of the Reynolds number on formation and topology of the vortices within the embedded cavity. The obtained results indicate that the cavity height is influential in the number of vortices. It is shown by increasing the Reynolds number, a single vortex tends to move towards the outlet. In addition, streamlines demonstrate that small vortices in vicinity of the cavity corner tend to be enlarged with increase of the Reynolds number. The developed numerical model can be extended to the flow structure of natural systems such as an embayment subjected to parallel-to-shore currents.
\end{abstract}

\section{INTRODUCTION}

Couette flow in fluid dynamics refers to the flow of a viscous fluid in the space between two parallel surfaces, one of which is moving tangentially relatively to the other. The configuration generally takes the form of two parallel plates or the gap between two concentric cylinders. Couette flow is driven by virtue of viscous drag force acting on the fluid, but it may additionally be motivated by an applied pressure gradient in the flow direction. The classical Couette flow problem under various conditions has been widely investigated [1-6].

In literature, several studies have been carried out to analyze the Couette flow problem in presence of cavities. However, in these studies far more attention has been devoted to the stability analysis of Couette flow [7,8] and less attempt has been made to analyze the vortex formation in the cavity due to Couette flow. Indeed, the phenomenon of vortex formation in cavities has many possible applications, particularly in natural and environmental sciences, such as the investigation of gyres within open lacustrine embayment [9]. In this context, the phenomenon of vortex formation in cavities has been comprehensively investigated, both experimentally and numerically [10-15]. For instance, Cheng at al. [14] employed the Lattice Boltzmann Method (LBM) to investigate the vortex structure in a rectangular cavity with various aspect ratios under different Reynolds number values. The effects of aspect ratio and

\footnotetext{
* Corresponding author: eugenia.rossidischio@unibo.it
} 
Reynolds numbers on size, center position and number of vortices were determined together with the flow pattern in the cavity. In another study [15], the Kármán vortex street inversion in the wake of a square cylinder under magnetic interaction has been studied with particular emphasis to the causes of the vortex inversion and to the physical mechanisms. It was concluded that the inversion phenomenon may be formed by controlling the relative narrow mean flow and the primary vortex with sufficient strength in the downstream.

Although the comportment of Couette flows in cavities have been widely studied, analyses of the vortex formation and topology in cavities due to Couette flow are relatively rare in literature. To fill this gap, the present study aims to investigate numerically formation and topology of vortices in Couette flow with low Reynolds numbers inside a rectangular duct with an embedded cavity. The effects of the cavity aspect ratio, namely ratio of the height to length of the cavity, and variations of the Reynolds number on formation and topology of the vortices are addressed by means of the finite element simulations.

\section{NUMERICAL MODEL}

The geometry under study, illustrated in Fig. 1, is the so-called Couette configuration, i.e. two parallel plates, one of which (upper) is moving relatively to the other with uniform velocity $U_{0}$. In Fig. $1, H$ represents the channel height while $L_{l}$ and $H_{l}$ are the cavity length and height, respectively. Furthermore, $L_{0}$ and $L_{2}$ are the horizontal dimensions of the channel. The duct has a variable cross-section and consists of the inlet, outlet and the cavity serving as a vortex promoter. It was assumed that a Newtonian fluid, with constant thermophysical properties, flows in the channel under the steady-state condition. The fluid flow is twodimensional with the velocity vector $\vec{U}=(U, V)$.

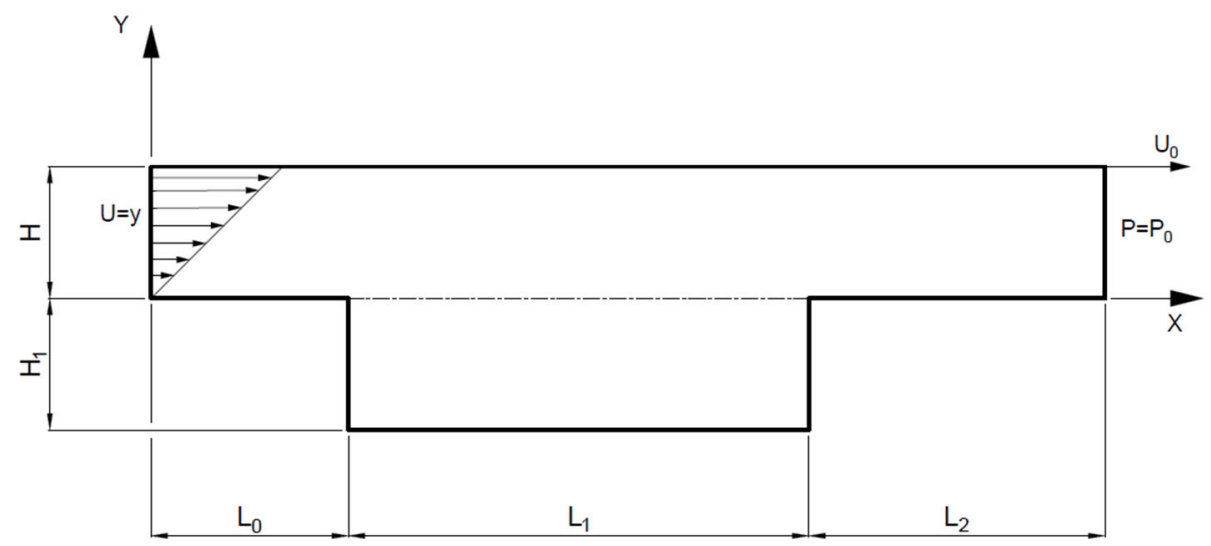

Fig. 1. A sketch of the domain under study. 
The governing equations, namely the mass and momentum equations, in dimensionless form are given by:

$$
\begin{gathered}
\frac{\partial u_{i}}{\partial x_{i}}=0 \\
u_{i} \frac{\partial u_{i}}{\partial x_{j}}=-\frac{\partial p}{\partial x_{i}}+\frac{1}{R e} \frac{\partial^{2} u_{i}}{\partial x_{i}^{2}}
\end{gathered}
$$

where the dimensionless variables are:

$$
\begin{gathered}
x_{i} \rightarrow(x, y)=\frac{(X, Y)}{H} \\
u_{i} \rightarrow(u, v)=\frac{(U, \mathrm{~V})}{U_{0}} \\
p=\frac{P-P_{0}}{\rho U_{0}^{2}} \\
\operatorname{Re}=\frac{U_{0} H}{v}
\end{gathered}
$$

where $v$ in Equation (6) is the kinematic viscosity of the fluid.

As a boundary condition, the no-slip condition was imposed at walls, i.e. zero velocity at the fluid-solid interface. At inlet, the velocity inlet was assumed given by the Couette velocity profile, namely $u=y, v=0$, while the pressure outlet given by $p=0$ was considered at outlet.

The variables in Fig. 1 are all dimensionless with respect to $H$ fixed at $1 \mathrm{~m}$.

To solve the mass and momentum balance equations, a 2D steady-state finite element code was developed through COMSOL Multiphysics environment. For each configuration, the computational domain was meshed with structured quadrilateral grid. Figure 2 displays the mesh employed for the case with $H_{1}=0.5$ and $L_{1}=1.5$, as well as details of the cavity corner.

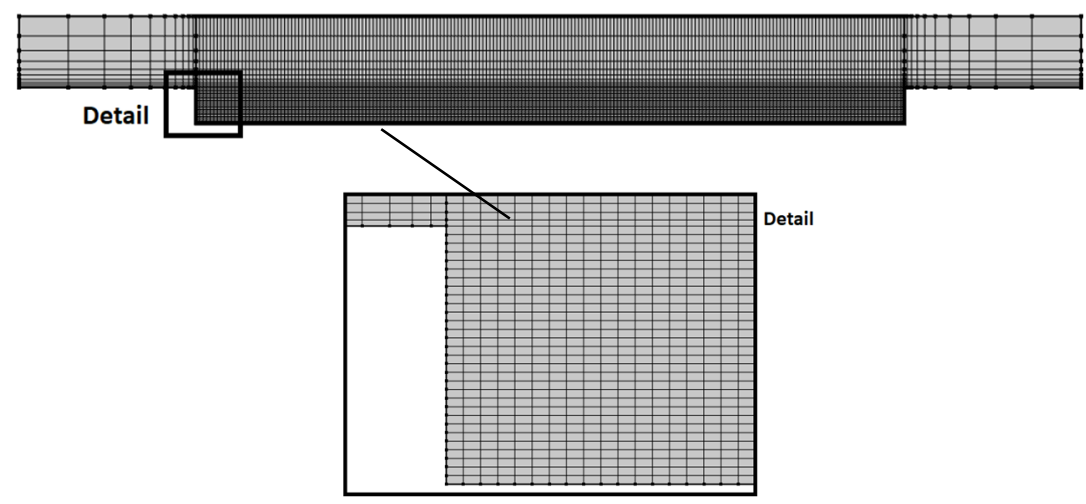

Fig. 2. A sample of the mesh employed and details of the cavity corner. 
The mesh has been constructed under a specific independence procedure. After fixing the control quantities, namely $u_{j}$ and $p_{j}$, defined as the velocity and pressure referred to the $j$-th mesh element, the relative errors are given by:

$$
\begin{aligned}
& \vartheta=\frac{\left|u_{j+1}-u_{j}\right|}{u_{j}} \times 100 \\
& \varphi=\frac{\left|p_{j+1}-p_{j}\right|}{p_{j}} \times 100
\end{aligned}
$$

where $\vartheta$ and $\varphi$ are the relative error indices referred to the velocity and to pressure, respectively.

Table 1. The grid independence test for $R e=500, H_{1}=0.5$ and $L_{1}=5$.

\begin{tabular}{ccccc}
\hline $\iint \boldsymbol{u} \boldsymbol{d} \boldsymbol{\Omega}$ & $\boldsymbol{\vartheta} \times \mathbf{1 0}^{-\mathbf{3}}$ & $\iint \boldsymbol{p} \boldsymbol{d} \boldsymbol{\Omega}$ & $\boldsymbol{\varphi} \times \mathbf{1 0}^{-\mathbf{2}}$ & $\begin{array}{c}\text { Number of } \\
\text { Elements }\end{array}$ \\
\hline 7,54649 & & 0,15883 & & \\
7,54796 & 0,1938 & 0,16284 & 0,02519 & \\
7,54873 & 0,1030 & 0,16423 & 0,8563 & \\
7,54921 & 6,304 & 0,16499 & 0,4600 & \\
7,54952 & 4,101 & 0,16546 & 0,2896 & \\
7,54963 & 1,522 & 0,16565 & 0,1116 & \\
$\mathbf{7 , 5 4 9 7 3}$ & $\mathbf{1 , 2 7 0}$ & $\mathbf{0 , 1 6 5 8 1}$ & $\mathbf{9 , 7 7 3}$ & $\mathbf{3 0 3 8 0 0}$ \\
7,54981 & 1,072 & 0,16595 & 8,750 & \\
\hline
\end{tabular}

Table 2. The grid independence test for $R e=500, H_{1}=10$ and $L_{1}=0.5$.

\begin{tabular}{ccccc}
\hline $\int \boldsymbol{u} \boldsymbol{d} \boldsymbol{\Omega}$ & $\boldsymbol{\vartheta} \times \mathbf{1 0}^{-3}$ & $\iint \boldsymbol{p} \boldsymbol{d} \boldsymbol{\Omega}$ & $\boldsymbol{\varphi} \times \mathbf{1 0}^{-\mathbf{2}}$ & $\begin{array}{c}\text { Number of } \\
\text { Elements }\end{array}$ \\
\hline 7,53912 & & 0,27245 & & \\
7,53922 & 0,1396 & 0,27221 & 8,886 & \\
7,53929 & 9,560 & 0,27200 & 7,751 & \\
7,53934 & 5,650 & 0,27188 & 4,368 & \\
7,53936 & 3,765 & 0,27178 & 3,686 & \\
7,53939 & 2,900 & 0,27171 & 2,397 & \\
$\mathbf{7 , 5 3 9 4 0}$ & $\mathbf{2 , 1 8 0}$ & $\mathbf{0 , 2 7 1 6 6}$ & $\mathbf{1 , 8 6 2}$ & $\mathbf{5 6 2 6 0 0}$ \\
7,53941 & 1,567 & 0,27162 & 1,501 & \\
\hline
\end{tabular}

For a given Reynolds number Re, several grid independence checks were performed. Here, for the sake of brevity two examples are presented in Table 1 and 2. Table 1 shows the grid construction procedure and independence test for a case with $R e=500, H_{1}=0.5$ and $L_{1}=5$ and Table 2 reports that for a case with $R e=500, H_{1}=10$ and $L_{1}=0.5$. The selected mesh for final computations in Table 1 and 2 has 303800 and 562600 elements, respectively. 


\section{RESULTS AND DISCUSSION}

The velocity contours at $R e=200$ for four different configurations are shown in Fig. 3 . As expected, the upper part of the channel has the highest velocity, due to tangential movement of the surface. The contours show that the fluid velocity in all configurations is a decreasing function of the channel height. In addition, figures indicate that the depth of the cavity is uninfluential in the fluid velocity distribution within the cavity, except of the very first centimeters of the cavity height. For instance, for the case with $H_{1}=2$ and $L_{1}=1.5$, the dimensionless fluid velocity takes an identical value within the cavity for the $H_{1}$ values lower than -0.5 . This trend can also be observed for other configurations.
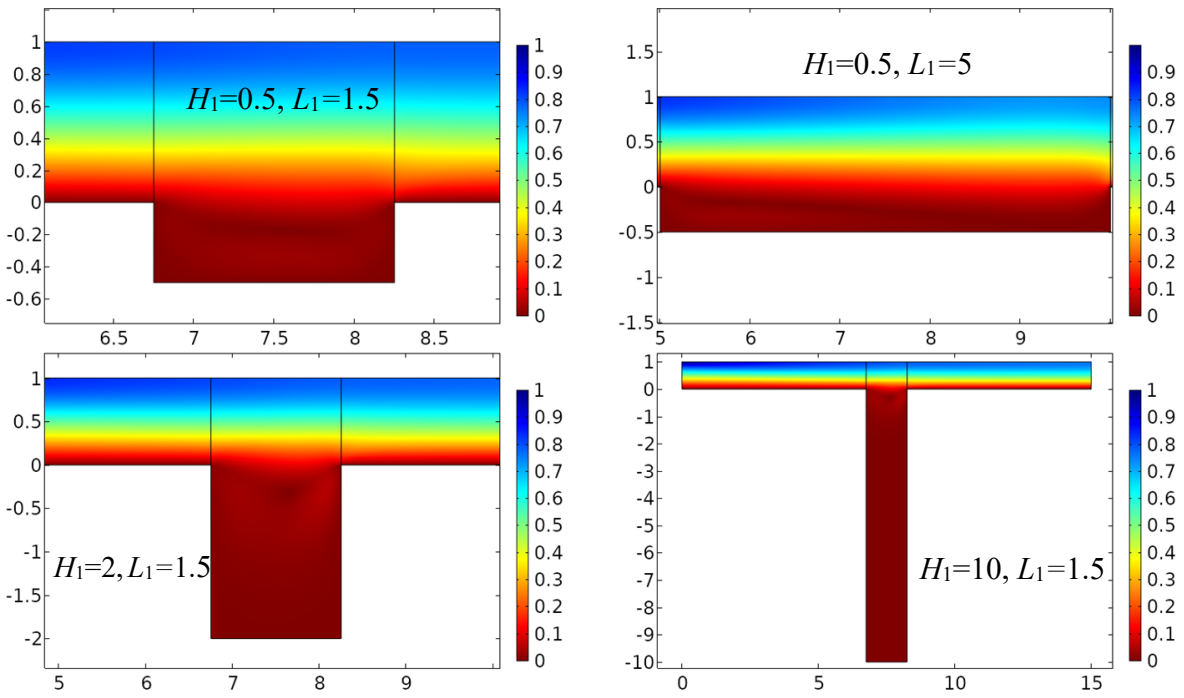

Fig. 3. Dimensionless velocity contours at $R e=200$ for different configurations.

Figures 4-7 illustrate the streamlines and the vortex formation for different Reynolds numbers under various configurations. In particular, Fig. 4 and 5 refer to the cavity with variable length and fixed height while Figs 6 and 7 represent the cavity having fixed length and variable height.

Figure 4 shows that, for a case with $H_{1}=0.5$ and $L_{1}=1.5$, the Reynolds number may affect the position of the vortex; as the Reynolds number increases, a single vortex tends to move its center towards the outlet. Streamlines of the Fig. 5 indicate that for a wide and shallow cavity, vortices are mainly formed in vicinity of the cavity corner. Moreover, it is noticeable that by increasing the Reynolds number, the length of the vortex increases.

Figure 6 shows that small vortices adjacent to the cavity corner can be enlarged with increase of the Reynolds number. It is evident here again that the center of the vortex tends to move towards the channel outlet when the Reynolds number increases. The similar trend can be seen in Fig. 7 where the cavity is relatively narrow and deep. Furthermore, the streamlines of Fig. 7 imply that the number of vortices depends on the cavity height. Nonetheless, the number of vortices is independent of the Reynolds number. 

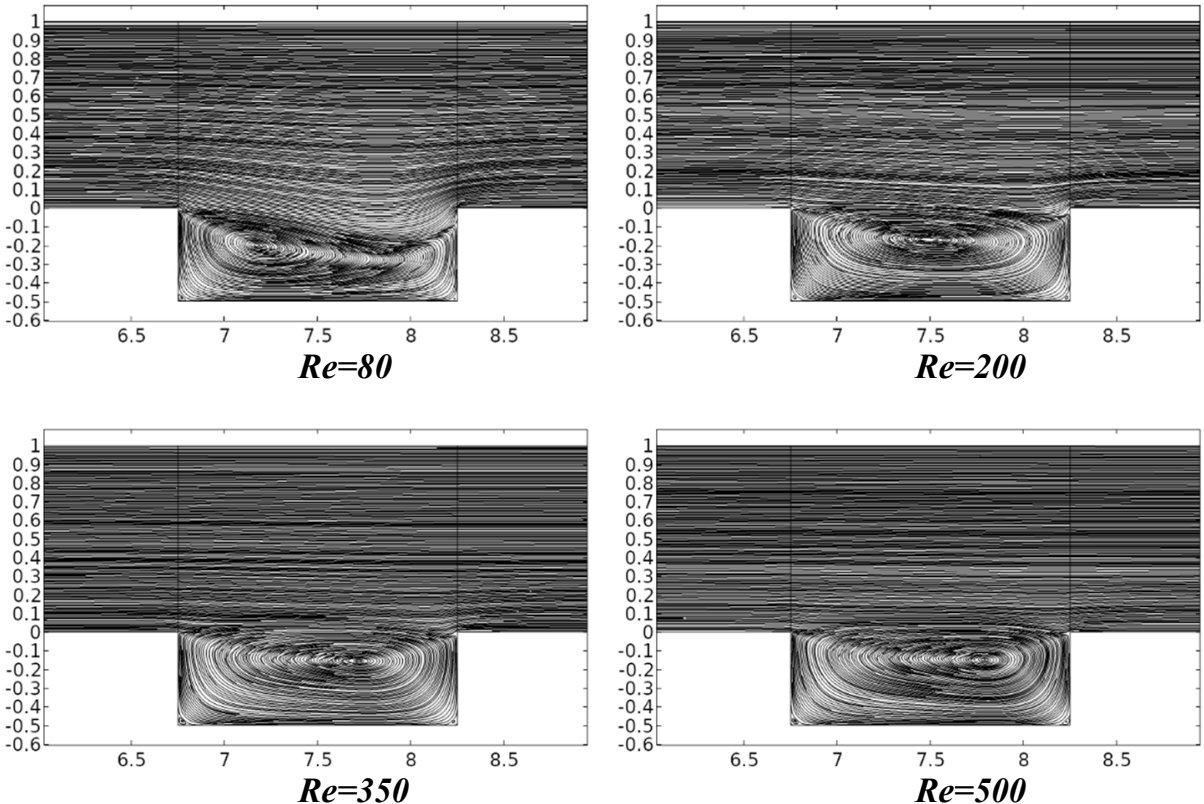

Fig. 4. Streamlines and vortex position for $H_{l}=0.5$ and $L_{l}=1.5$.
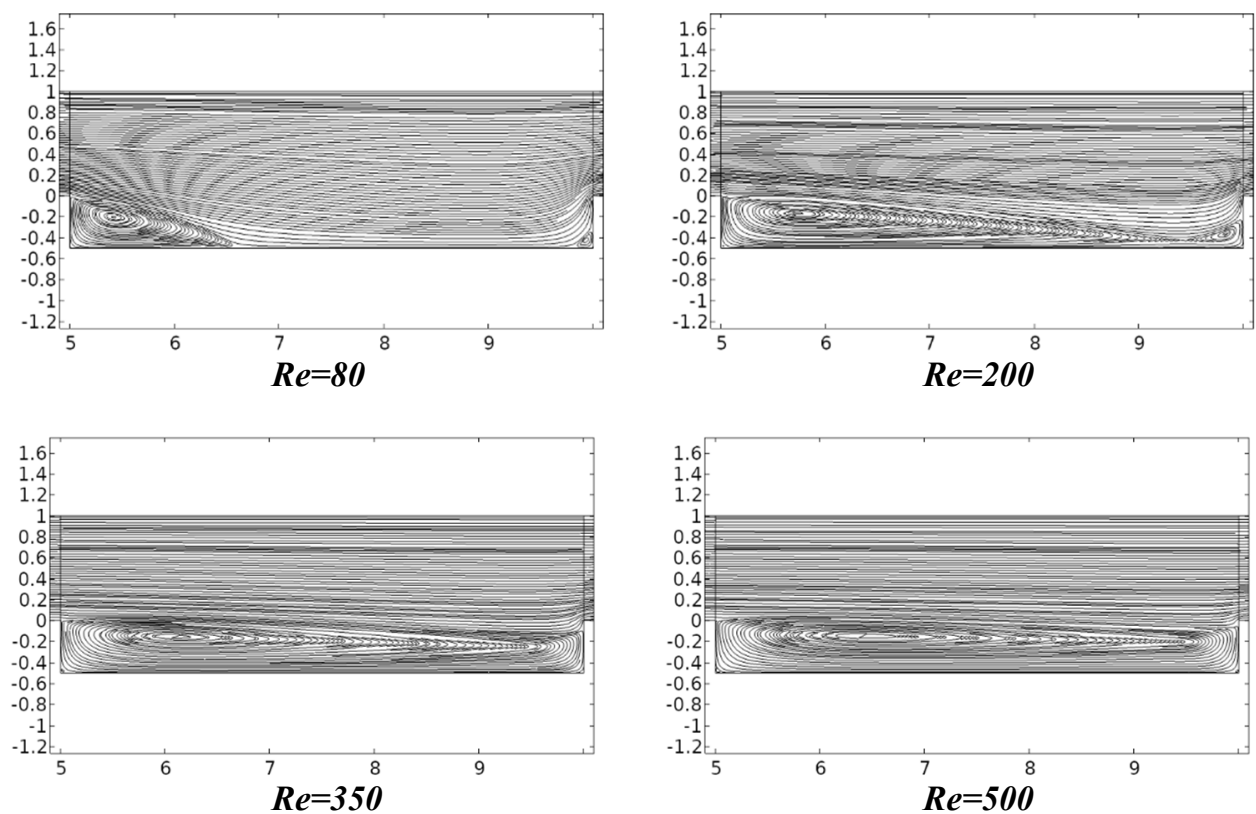

Fig. 5. Streamlines and vortex position for $H_{I}=0.5$ and $L_{I}=10$. 

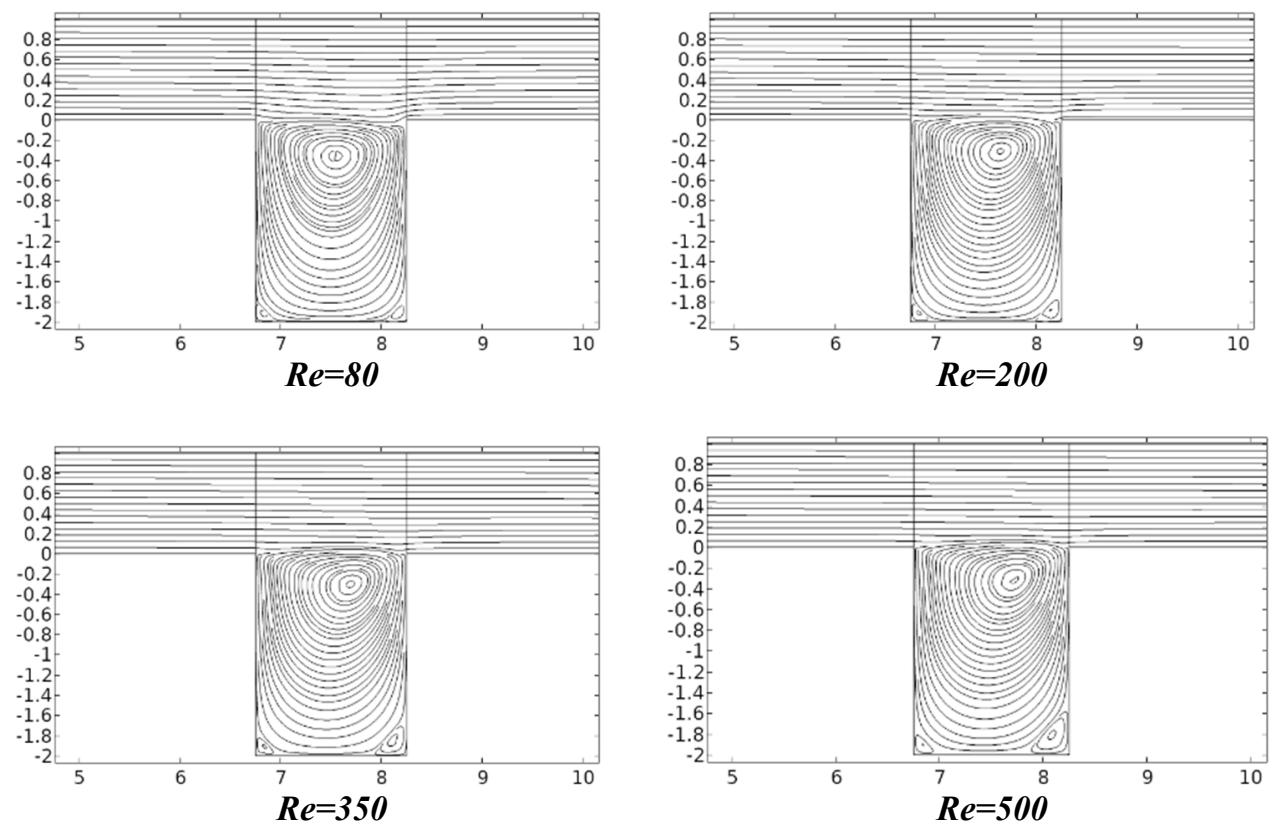

Fig. 6. Streamlines and vortex position for $H_{l}=2$ and $L_{l}=1.5$.
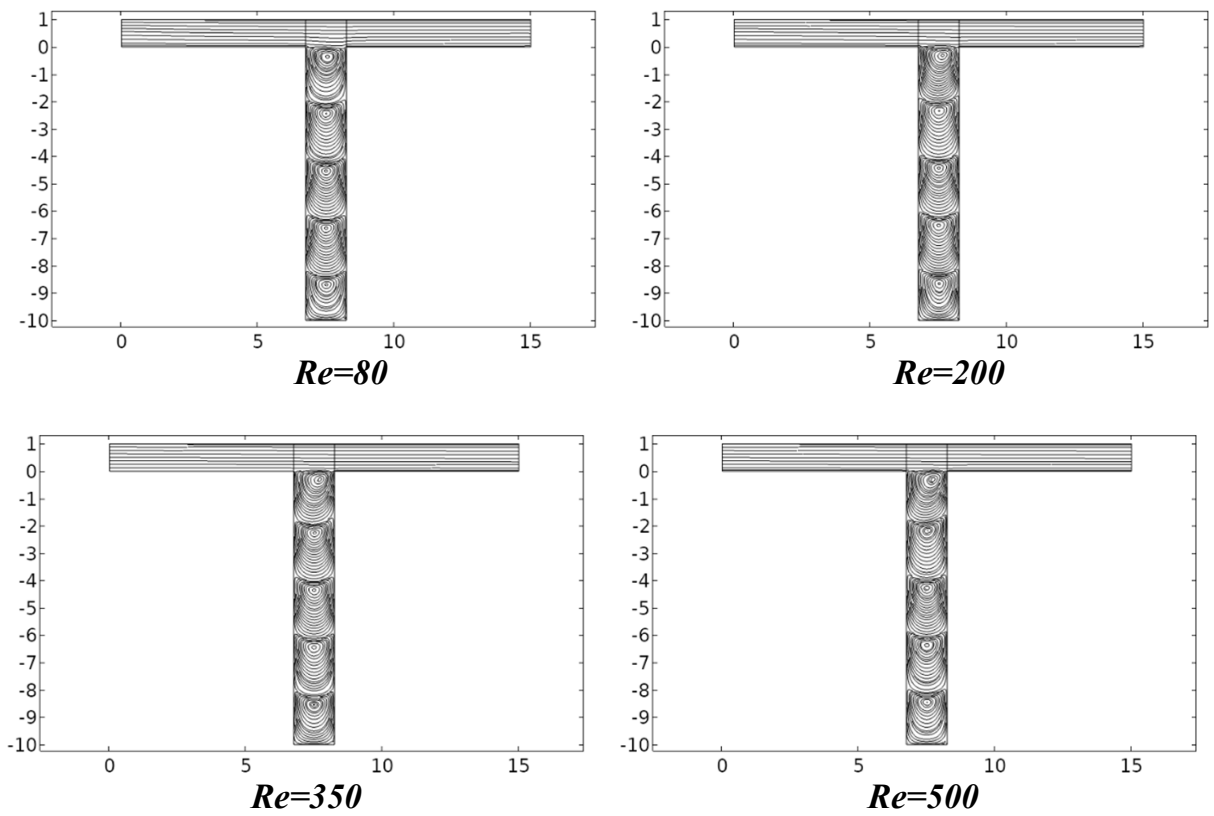

Fig. 7. Streamlines and vortex position for $H_{1}=10$ and $L_{1}=1.5$. 
In order to analyze the topology of vortices more precisely, positions of the vortex center for different values of the Reynolds number are investigated in a single frame. Figures 8 and 9 illustrate the vortex center for two opposite configurations, namely $H_{1}=0.5 \mathrm{~m}$ and $L_{l}=5 \mathrm{~m}$ and $H_{1}=5 \mathrm{~m}$ and $L_{1}=0.5 \mathrm{~m}$, for Various Reynolds numbers varying from 80 to 500 .

Figure 8 clearly shows for a rather wide and shallow cavity, with the augmentation of the Reynolds number, the vortex positioned close to the inlet section tends to grow and move towards the outlet section, until it is affected by the neighbor vortex. On the other hand, Fig. 9 shows that this effect is minimized for a rather deep and narrow cavity. Moreover, Fig. 9 represents that the vortex center moves slightly upwards when the Reynolds number increases.

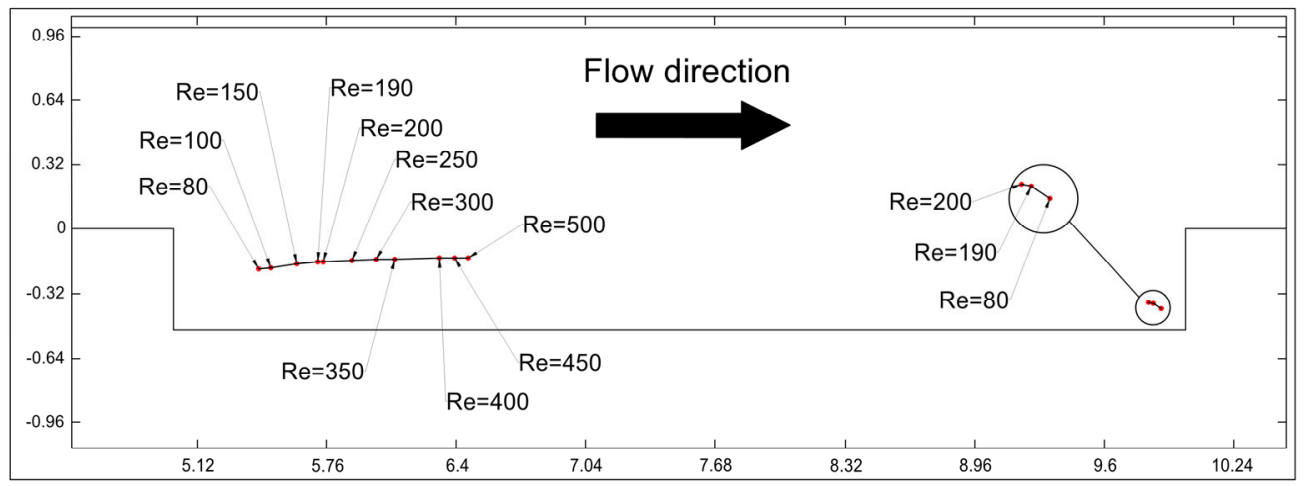

Fig. 8. The topology of the vortex center, for $H_{1}=0.5$ and $L_{1}=5$.

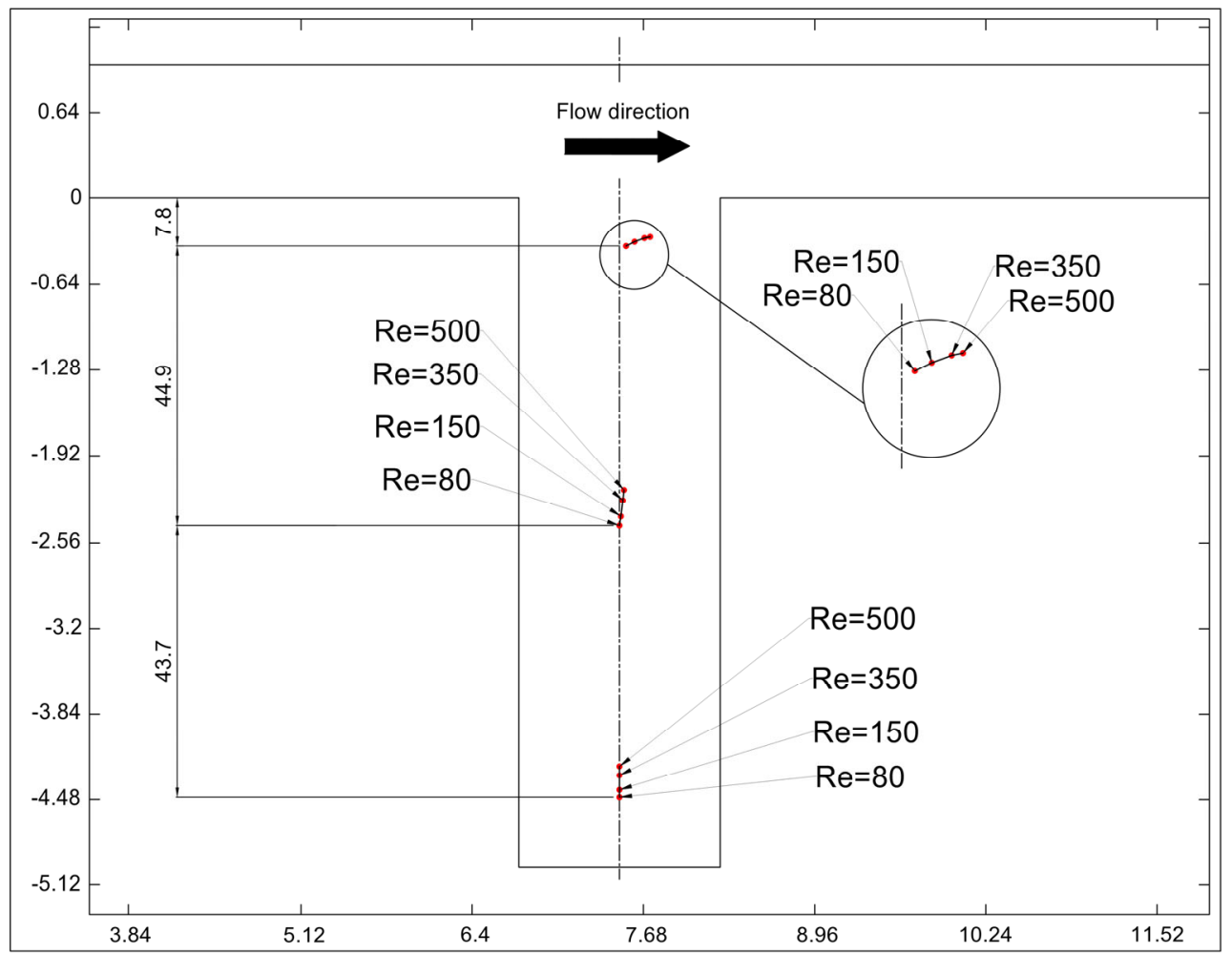

Fig. 9. The topology of the vortex center, for $H_{l}=5$ and $L_{l}=0.5$. 


\section{CONCLUSIONS}

The present study has sought to analyze the planar Couette flow in low Reynolds numbers applied to a channel with a morphing cavity serving as a vortex formation promoter. By employing a finite element code implemented in COMSOL Multiphysics, the effects of the aspect ratio of the cavity and the Reynolds number on formation and topology of the vortices have been investigated.

The obtained results have indicated that the cavity height affects the number of vortices. It has been shown that as the Reynolds number increases, a single vortex tends to move its center towards the outlet. Furthermore, streamlines within the cavity has demonstrated with increase of the Reynolds number, small vortices in vicinity of the cavity corner tend to be enlarged.

As a potential direction for future research, the presented numerical model can be employed and extended in order to analyze features of the several Couette configuration models in natural systems such as an embayment subjected to parallel-to-shore currents.

\section{References}

1. A. Barletta, E. Magyari, Int. J. Therm. Sci., 47(7), 811-819 (2008).

2. A. Abramov, A. Butkovskii, Int. J. Heat Mass Transf., 117, 313-318 (2018).

3. M.A. Rana, Y. Ali, B. Ahmad, M.T.A. Rana, Coatings, 9, 553 (2019).

4. C. Tantos, Eur. J. Mech. / B Fluids , 76, 375-389 (2019).

5. Y. Damianou, P. Panaseti, G. Georgiou, Materials, 12, 3574 (2019).

6. L. Mirantsev, A. Abramyan, Phys. Lett. A, 384, 126181 (2020).

7. G. A. Bres, T. Colonius, J. Fluid Mech., 599, 309-339 (2008).

8. E. Serre, M. A. Sprague, R. M. Lueptow, Phys. Fluids, 20(3) 034106, (2008).

9. A. M. Razmi, U. Lemmin, D. Bouffard, A. Wüest, R. Uittenbogaard, D. A. Barry, Environ. Fluid Mech., 17(3), 415-428 (2017).

10. S. Taneda, J. Phys. Soc. Japan, 46(6), 1935-1942 (1979).

11. C. Shen, J. Floryan, Phys. Fluids, 28(11), 3191-3202 (1985).

12. K. Gustafson, K. Halasi, J. Comp. Phys.ics, 64(2), 279-319 (1986).

13. M. Rosenfeld, E. Rambod, M. Gharib, J. Fluid Mech., 376, 297-318 (1998).

14. M. Cheng, K. Hung, Comp. Fluids, 35(10), 1046-1062 (2006).

15. L. Chen, S.-J. Xu, M.-J. Ni, Int. J. Heat Mass Transf., 117, 768-779 (2018). 